\title{
Alfonso López Arroyo: pionero de la Sismología y la Ingeniería Sísmica en España
}

\author{
ANTONI ROCA ADROVER ${ }^{1}$, JOSE MANUEL MARTÍNEZ SOLARES ${ }^{2,3}$ \\ \& AGUSTIN UdíAs VALLINA ${ }^{3}$ \\ ${ }_{2}^{1}$ Institut Geològic de Catalunya \\ ${ }^{2}$ Instituto Geográfico Nacional \\ ${ }^{3}$ Dpto. Geofísica y Meteorología, Universidad Complutense \\ aroca@igc.cat
}

Recibido: $10 / 05 / 2012$

Aceptado: 05/09/2012

\begin{abstract}
Resumen
En su trayectoria científica y profesional, Alfonso López Arroyo, uno de los pioneros de la Sismología y la Ingeniería Sísmica en España, ha tratado múltiples temas, en particular aquellos que impliquen la interrelación entre diferentes campos de las Ciencias de la Tierra y la Ingeniería, en los cuales ha realizado importantes contribuciones. Aunque su actividad no se desarrolló fundamentalmente en el área de la docencia, su labor de enseñanza e influencia en muchos científicos y profesionales españoles es indiscutible.
\end{abstract}

Palabras clave: Biografía, geofísica, sismología, ingeniería sísmica.

\section{Alfonso López Arroyo: Pioneer of the Seismology and the Earthquake} Engineering in Spain

\begin{abstract}
In his scientific and professional career, Alfonso López Arroyo, one of the pioneers of Seismology and Earthquake Engineering in Spain, has been devoted to multiple topics, particularly those involving interrelation between different fields of Earth Sciences and Engineering, in which he made important contributions. Even though his activities have not been mainly developed in the field of university teaching, his educational role and his influence in many scientists and professionals is unquestionable.
\end{abstract}

Keywords: Biography, geophysics, seismology, earthquake engineering.

Sumario: Introducción. 1. Trayectoria académica y profesional. 2. Trayectoria científica. 3. Interacción con grupos de trabajo multidisciplinarios y transferencia de conocimiento. 4. Anexo: Bibliografía seleccionada de Alfonso López Arroyo.

\section{Referencia normalizada}

Roca Adrover, A., Martinez Solares, J.M. \& Udias Vallina, A. (2012). Alfonso López Arroyo: pionero de la Sismología y la Ingeniería Sísmica en España. Física de la Tierra, vol. 24, 11-16.

\section{Introducción}

El terremoto de Lorca del 11 de mayo de 2011 ha puesto de manifiesto la vulnerabilidad de la sociedad ante un sismo de moderada magnitud, causando importantes pérdidas económicas y algunas víctimas humanas. Lo más constatable, sin 
embargo, es la todavía importante necesidad de disponer de las herramientas adecuadas para la realización de evaluaciones de soporte a la gestión de la emergencia. Evaluaciones que requieren la contribución del conocimiento de disciplinas diversas.

Después del terremoto, como aparece en este volumen monográfico, un número considerable de científicos y profesionales están realizando estudios y trabajos de diferentes ámbitos de las ciencias de la Tierra e ingeniería: sismología, geodesia, geología, ingeniería, arquitectura, técnicas de gestión de emergencias, ... resaltando la necesidad de comunicación y colaboración en tareas multi e interdisciplinarias.

El panorama actual - en cuanto al número de equipos trabajando en estas materias - es muy diferente del que existía en España a finales de los años 60 y comienzos de los 70 del siglo pasado. Podemos afirmar que en aquellos momentos tan solo había tres sismólogos con relevante proyección internacional: Alfonso López Arroyo y Gonzalo Payo Subiza, del Instituto Geográfico Nacional (IGN) en aquel momento Instituto Geográfico y Catastral - y Agustín Udías Vallina, que se incorporaría a principios de los 70 a la Universidad Complutense, después de unos años en la Universidad de Barcelona y manteniendo también una estrecha colaboración con el IGN. Los tres realizaron importantes trabajos, algunos de forma conjunta, que constituyeron la base de la moderna sismología en España.

Alfonso López Arroyo trabajó en muchas de estas disciplinas aplicadas al terremoto de Lorca, despertando el interés, transmitiendo sus conocimientos, formando y dando su siempre valioso apoyo a muchos de los investigadores y profesionales que actualmente dirigen e integran muchos de los grupos que han estado trabajando en este terremoto de Lorca.

De ahí lo oportuno por parte de los editores de este volumen de dedicarlo a Alfonso López Arroyo, en reconocimiento de su labor y contribución, muchas veces callada y siempre discreta, al estudio de los terremotos.

\section{Trayectoria académica y profesional}

Alfonso López Arroyo se licenció en Ciencias Matemáticas en la Universidad de Madrid el año 1950, realizando los cursos de doctorado en Ciencias Matemáticas y Físicas en la misma universidad en los años 1952 y 1953.

En 1954 obtuvo el Master of Science en Geofísica por la Universidad de Saint Louis, Missouri, Estados Unidos de Norteamérica. En 1956 es Ingeniero Geógrafo en el Instituto Geográfico y Catastral (actualmente Instituto Geográfico Nacional, IGN) y en 1961 Doctor Ingeniero Geógrafo.

Su larga trayectoria profesional e investigadora comienza el año 1951 como becario del Instituto Jorge Juan de Matemáticas del Consejo Superior de Investigaciones Científicas (CSIC) y el 1953 en el Instituto Nacional de Geofísica del CSIC. El curso1952-53 es Profesor Ayudante en la Universidad de Madrid, y en los cursos 1953-54 y 1955-56 es Profesor Adjunto en la misma universidad. Entre 1955 y 1958 es Ayudante de Investigación del Instituto Nacional de Geofísica, instituto del cual es Colaborador Honorario desde el año 1958. 
En el IGN, entre 1958 y 1965 es director del Observatorio Sismológico de Málaga, pasando a ocupar los cargos de Jefe del Departamento de Investigación entre 1966 y 1970; Jefe del Servicio de Sismología (1970-72); Jefe del Departamento de Geofísica del Centro de estudios Geográficos, Geofísicos y Astronómicos (1972-74); Jefe de la Sección de Sismología e Ingeniería Sísmica (1974-79); Jefe del Servicio de Geofísica (1979-80). En 1980 es nombrado Subdirector General de Geodesia y Geofísica. Al cesar en este puesto ocupa la Jefatura del Área de Geofísica hasta su jubilación como funcionario en 1992.

Durante su vida profesional realiza estancias en diversos países, colaborando con centros de reconocido prestigio como la universidad de Upsala, donde es Investigador Asociado durante dos períodos de tres meses cada uno en 1961 y 1963, y en el Laboratorio de Investigación Sísmica, Servicio Geológico de los Estados Unidos (USGS) en Boulder, Colorado donde es Investigador Asociado, senior, (1972-73).

También ha desarrollado sus actividades profesionales en compañías privadas. Desde 1956 a 1958 fue Jefe de Equipo y posteriormente hasta 1960 Asesor Técnico en Geophysical Prospecting Co. Más tarde fue también Asesor Técnico de Hispanoil (1965-67) y de Unión Explosivos Rio Tinto (1970-72). Ha colaborado en diversos proyectos con grupos de trabajo de diversos organismos públicos y privados: Universidad Complutense, Ofiteco, Servicio Geológico de Cataluña,...

Alfonso López Arroyo

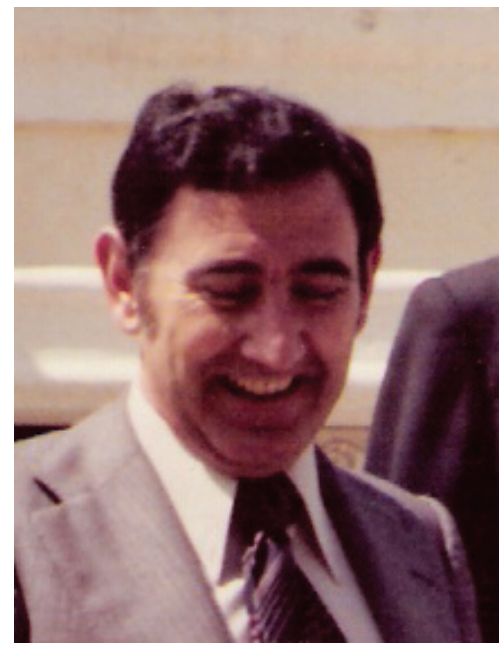

\section{Trayectoria científica}

En un momento en que las contribuciones de autores españoles en revistas del Science Citation Index era muy escaso, y prácticamente nulo en el campo de las Ciencias de la Tierra, Alfonso López Arroyo, ya entre el año 1962 y 1976 publicó 
en revistas de impacto como el Journal of Geophysical Research (JGR), Pure and Applied Geophysics (PAGEOPH), Tectonophysics, Bulletin of the Seismological Society of America (BSSA), entre otras. Cabe destacar incluso su publicación, el año 1972, con Agustín Udías en NATURE: "Plate tectonics and the AzoresGibraltar región", una temprana publicación sobre tectónica de placas, en un momento en el que en muchas de las universidades, en los estudios de geología todavía no se explicaba esta teoría.

\section{Interacción con grupos trabajo multidisciplinarios y transferencia de conocimiento.}

A lo largo de su dilatada actividad profesional ha formado parte y ha tenido funciones relevantes en múltiples Comisiones y Asociaciones científicas y profesionales nacionales e internacionales. Ha sido Presidente de la Sección de Sismología y Física del Interior de la Tierra de la Comisión Nacional de Geodesia y Geofísica, Secretario de la Comisión Permanente de Normas Sismorresistentes, Vicepresidente de la Subcomisión de Sismicidad de la Comisión Sismológica Europea, representante español y miembro de diversas comisiones científicas y Presidente de la Asociación Española de Ingeniería Sísmica desde 1977 hasta 1995. Asimismo, es Vocal de la Junta Directiva de la Real Sociedad Geográfica y Académico Correspondiente de la Real Academia de Doctores de España.

Ha sido miembro de diversos grupos de expertos, entre ellos el de movimientos del suelo de Comité de Seguridad de instalaciones nucleares, OCDE o el grupo de trabajo de Investigación de la Tierra de la Agencia Espacial Europea. Fue uno de los creadores del Grupo de Trabajo Ibero-Mogrebí.

Conocedor e impulsor de sistemas instrumentales de vigilancia sísmica colaboró activamente con su imprescindible asesoramiento en proyectos relacionados con presas y centrales nucleares.

Ha participado en la organización de un número importante de congresos, conferencias y workshops. Entre ellos cabe destacar la co-organización del Congreso Mundial de Ingeniería Sísmica (10WCEE), un macro-evento celebrado en Madrid el año 1992.

Alfonso López Arroyo ha cultivado diferentes campos dentro de las ciencias de la Tierra y siempre se ha sentido cómodo en actividades inter y multidisciplinarias. Ha fomentado temáticas "frontera": sismología - ingeniería, sismología técnicas geofísicas, ... y ha colaborado con diferentes grupos en ámbitos como los estudios de vulnerabilidad y riesgo, acciones de transferencia de conocimiento centros de investigación - administración - empresa, y aspectos de protección civil, entre otras.

Su inteligencia, su agudeza, su amplio espectro de conocimientos y experiencia, su espíritu de colaboración, su capacidad de intuición, su vocación de constante innovación, su generosidad y su carácter afable han sido siempre fuerzas incentivadoras para los equipos con los que ha trabajado. Sus aportaciones han influido de forma altamente positiva en muchos de sus colaboradores que lo 
reconocemos como un maestro fundamental en nuestra formación científica, técnica y profesional.

\section{Anexo: Bibliografía seleccionada de Alfonso López Arroyo}

Las contribuciones de Alfonso López Arroyo en revistas nacionales e internacionales, presentaciones en congresos y monografias técnicas son numerosas. Hemos realizado una selección en base a diversos criterios: bien porque se trate de sus artículos más relevantes en revistas de prestigio, bien para mostrar la variedad de temas a los que se ha dedicado, o bien para resaltar el carácter pionero de sus actividades.

- Cálculo de los errores cometidos en las reducciones topográfica e isostática de la gravedad, Rev. de Geofísica, no 42, 173 - 178, 1952.

- Mareas terrestres, Rev. de Geofísica, no 49, 37 - 76, 1954.

- Acerca de la anisotropía elástica de la corteza terrestre, Rev. de Geofisica n $^{\circ}$ $50,169-173,1954$.

- Attenuation and dispersión of G-waves, en colaboración con M. Bath, $J$. Geophys, Res. V 67, n 5, 1933-1942, 1962.

- Pa and Sa waves and the Upper Mantle, en colaboración con M. Bath, Geof. Pura e appl. (PAGEOPH) V. 56, 57 - 92, 1963.

- Background noise on the SSS standard instruments, Scientific Report No. 4, VELA-UNIFORM project, 31 pp. + 13 fig. 1964.

- Crustal Structure of the Spanish Central Plateau, en colaboración con G. Payo, Proc., IX Gen. Ass. European Seismological Commission, 77-86, 1966.

- Recording capabilities of some Spanish seismological stations, Scientific Report No. 21, VELA-UNIFORM project, 8 pp. +6 fig. 1967.

- Body and surface wave study of source parameters of the 15 March 1964 Spanish earthquake, en colaboración con A. Udías, Tectonophysics, v. 9, nº 1 , 323-346, 1970.

- Aftershock sequence and focal parameters of the February 28, 1969 earthquake of the Azores-Gibraltar fracture zone, en colaboración con A. Udías, Bull, Seim. Soc. of Am. v. 62 no 3, 699-720, 1972.

- Curvas medias de variación de la intensidad sísmica con la distancia epicentral en España, en colaboración con D. Muñoz Sobrino y J. Mezcua, Com. 1 Asamblea Nac. Geod. y Geof., t.I. 327-340, 1974.

- Deterministic and probabilistic approach to determine seismic risk of nuclear power plants; a practical example, en colaboración con A. Soriano y J.M. Roesset, Proc. Symp. Earthq. Risk for nuclear power plants, Walferdange, 7987, 1975.

- Sismicidad inducida por embalses; métodos de estudio y sistemas de vigilancia, en colaboración con A. Pérez Saiz, Rev. Obras Públicas, nº Sept. y Oct., $26 \mathrm{p}, .1975$.

- Seismotectonics of the Azores - Alboran region, en colaboración con A. Udías y J. Mezcua, Tectonophysics, v. 31, 259-289, 1976. 
- Los terremotos ocurridos en Friuli (Italia) en 1976, en colaboración con J. Villacañas y A. Garcia Yagüe, Comisión Permanente de Normas Sismorresistentes, Presidencia del Gobierno, 46 pp +76 fig. 1977.

- Deterministic and probabilistic approaches to seismic risk determinations, en colaboración con A.F. Espinosa, Proc. Symp. Analysis of Seismicity and on Seismic Risk, Liblice, 487-496, 1977.

- Earthquake instrumental intensity from strong ground motions, en colaboración con A.F. Espinosa, Proc. VI World Conf. on Earthquake Engineering, v. $7,367-374,1977$.

- Isoseismal map of the 1755 Lisbon earthquake obtained from Spanish data, en colaboración con J. M. Martínez Solares y J. Mezcua, Tectonophysics, v. 53, $301-313,1979$.

- Some problems related to the seismic design of dams located in region with moderate seismicity, en colaboración con A. Roca y A. Soriano. Proc. 13 ème Congrès des Grandes Barrages, New Delhi, Q.15, R.35, 1447-1459, 1979.

- Sismicidad, agitabilidad y riesgo sísmico, Publicación técnica $\mathrm{n}^{\circ} 8$, IGN, 1979.

- Influencia de las condiciones del terreno y del tipo de construcción sobre los efectos del terremoto de Andalucía de 1884, en colaboración con A. J. Martín y J. Mezcua, Publicación técnica del IGN, 1981.

- Application of the Gumbel III law to seismic data from Southern Spain, en colaboración con A. Roca y E. Suriñach, Engineering Geology, 20, 63-71, 1984.

- The first accelerograms obtained in Spain. Soil and Source effects, en colaboración con A. Roca y G. Sánchez, Proc. IX World Conference on Earthquake Engineering, Tokyo y Kyoto, 8, 169-174, 1988.

- Early seismographic instruments in Spain, en colaboración con J. Cruz, A. Roca y C. Olivera En: Gli strumenti sismici storici, Italia e contesto europeo. Ferrari, G. (editor). SGA Storia-Geofisica-Ambiente, Bolonia, 161-164, 1990.

- Metodología Simplificada para el Analisis del Riesgo Sísmico, en colaboración con J. Villacañas, Rev. Física de la Tierra, v. 11, 269-284, 1999.

- The great historical 1755 earthquake. Effects and damage in Spain, en colaboración con J.M. Martinez Solares, Journal of Seismology, v. 8, 275-294, 2004.

- O terramoto de 1755 em Espaha, en colaboración con J.M. Martinez Solares, en $O$ grande Terramoto de Lisboa, vol. 1, cap. VII, 237-264. Fundaçao Luso Americana, 2005. 